Fecha de recepción: abril 2020

Fecha de aceptación: mayo 2020

Versión final: junio 2020

\section{El objeto de planchar. El Diseño entre la industria y la cultura social. Una reflexión ecosófica}

Alan Neumarkt ${ }^{(1)}$

\begin{abstract}
Resumen El dilema del Diseño Industrial está en su origen y en su relación directa como engranaje de la maquinaria industrial. Un ejemplo analizado (la plancha) sirve como referencia para comprender una primer fase cultural, una siguiente de desarrollo y una reciente como política de progreso, lo que implica consumo: energético y económico. Diecisiete inexplicables siglos sin cuestionar algo tan elemental.

La profesión podrá intentar ir hacia un camino de sustentabilidad, pero deberá hacerlo en paralelo al de la propia sociedad, o no tendrá poder de imponerse. "Estamos amenazados por nuestra propia tecnología”, plantea Andrew Feenberg. El Diseño Industrial debe generar su propia respuesta.
\end{abstract}

Palabras claves: Diseño - Industria - Cultura - Ecosofía.

[Resúmenes en inglés y portugués en la página 229]

(1) Diseñador Industrial (UNLP). Doctor en Diseño (UBA). Profesor Titular Regular (UNMdP). Profesor Titular Posgrado (UBA / UNTreF). Director de Educación Superior (ORT Argentina).

En memoria de Frank Memelsdorff, pionero del

Diseño Industrial Argentino.

El diseño sustentable es hoy un término incorporado al discurso académico disciplinar y este texto hará una reflexión al respecto, específicamente sobre el consumo de energía de productos hogareños, sus razones culturales y políticas, y su vinculación a la historia de la profesión en Argentina.

El diseño industrial -en tanto disciplina transversal- ha sido analizado en múltiples planos. En algunos niveles con mucha profundidad. Podemos nombrar la visión desde la semántica del objeto (Jung, Krippendorff) o también desde la filosofía de la técnica (Marcuse, Peirce, Simondon, Sennett). Hay textos que coinciden con el avance tecno-cultural, otros se oponen. Mientras tanto, el mundo sigue girando. 
La sustentabilidad como ítem proyectual en diseño industrial apenas cuenta con unos 35 años de aplicación, en lo académico y en la industria y -en opinión de quien escribe- sin profundizar totalmente el concepto. Hasta entrados los años ochenta del siglo veinte, no era un tema en discusión. En algunos casos se coloca la mirada solamente en el producto, sin considerar todo el proceso de fabricación. A veces se habla de la producción industrial sin ahondar demasiado en el cómo, ni en el porqué de determinadas prácticas. Se acepta al Industrialismo como adecuado, se generan actividades cotidianas y convivimos en una cultura diaria consumista sin demasiada explicación. O mejor dicho, con algunas explicaciones económicas y no tantas en lo cultural o antropológico.

Existen actualmente algunos libros de objetos Green Design, con un mayor foco en la vida útil del producto o pensados para un futuro reciclado y muy poco se dice del consumo de energía, ni de cómo se llegó hasta el punto actual, donde el uso de energía hogareño está en ascenso (Evans, 2017). En sólo dos generaciones hemos pasado de conservar alimentos en refrigeradores con barras de hielo a tener heladeras con freezer no frost; de calentar agua con carbón de leña a consumir gas butano de red a costos relativamente bajos; de iluminar con velas a transmitir televisión las 24 horas del día. De pasar de más de veinticinco siglos transportándonos con caballos, a los últimos cien años consumiendo combustibles fósiles no renovables. Damos por sentado el automóvil -como función, como signo de urbanidad y como status social- cuando en términos históricos sólo atravesaron un siglo, y muchas dudas hay sobre su futuro.

A diferencia de la Arquitectura, cuya renovación es muy lenta y la permanencia de los edificios suele superar la generación que lo construyó, en los productos industriales de consumo -haciendo referencia a los pertenecientes al espectro del diseño industrial- la renovación es muy alta, la vida útil relativamente programada, los volúmenes de producción permiten amortizaciones de costo, y la evolución tecnológica y de materiales incide rápidamente en la producción.

Las universidades enseñan Diseño Industrial desde el plano visual y su gramática. La semántica, la filosofía y comprender la sustentabilidad, incluyendo el proceso industrial completo requieren estudios avanzados, materias específicas o posgrados. Hay un territorio académico aún muy inexplorado. La industria solamente considera lo que mejora la rentabilidad; la academia apenas incorpora estas áreas a la enseñanza. Mientras tanto la sociedad -y sus tensiones- van generando la legislación pertinente y la cultura de consumo. Un seminario dictado hace unos años por el Dr. Ricardo Gómez profundizó en las nociones de Ecosofía y en las lecturas de Andrew Feenberg con respecto a la tecnología y la sociedad:

La necesidad de abandonar el paradigma dominante así como la necesidad de una nueva filosofía abarcadora para discutir, evaluar y proponer cambios en las prácticas y políticas necesariamente democráticas sobre el uso y preservación del medio ambiente (Feenberg, 2010; Gómez, 2015). 


\section{La industrialización}

Se podría comenzar desde los inicios del hábitat humano con las conductas vinculadas a los objetos. El Dr. Arq. Doberti -profundizando una idea que ya había relatado Sottsass en los años setenta del siglo veinte- describe con exquisita prosa los inicios del uso de los utensilios: "Entonces, alguien distinguió ( ) entre la Materia y la Forma, reconoció por un lado la resistencia y el peso del sílex, y por otro, la configuración acuñada que proveía el filo ( )" (Doberti, 2011).

Sin ahondar en casi toda la historia de la humanidad a partir de ese punto inicial se puede afirmar que, no habría Diseño Industrial sin Industria. Podría haber objetos no industrializados, algunos productos de algún mayor o menor grado de artesanía o producción, artes decorativas, entre otros. En esto, la historia está repleta de cosas. Pero para que verdaderamente consideremos a los objetos producidos dentro del campo del Diseño Industrial debe haber Industria. Y la Industria tiene su origen con su propia Revolución. En el siglo XVIII, en el Imperio Británico.

Tratar de explicar un fenómeno como la Revolución Industrial requiere más de un libro. Como menciona Ashton:

( ) hasta la exactitud de la denominación es discutible. Los cambios producidos en Inglaterra, Gales y Escocia no fueron sólo industriales, sino también sociales, económicos e intelectuales. Entre 1760 y 1830 las aldeas se convirtieron en ciudades, se tendieron las vías férreas para las nuevas locomotoras, se desarrollaron nuevas materias primas, el dinero comenzó a circular con fluidez, nació el sistema bancario, muchos viejos privilegios y monopolios fueron barridos y se fomentó la libre empresa. Ideas innovadoras y progresistas transformaron la vida (Ashton, 1959).

La razón y la máquina cambiaron al mundo. Y la industria cambió el medio ambiente, el uso de energía y -como puede ya verse en este siglo- alteró el clima. Tal vez de forma irreversible.

Se debe mencionar aquí a un hombre fundamental, investigador y mecánico en la Universidad de Glasgow -ensimismado y soñador, dice Klinckowstroem en su Historia de la Técnica- el más grande inventor de su época: James Watt (1736-1819). Watt logró el perfeccionamiento de la máquina de vapor de Newcomen. La historia premió a Watt designando con su apellido a la unidad de potencia, y por su aporte al Imperio Británico fue honrado y sepultado en la Abadía de Westminster junto a la realeza.

Entonces se podría decir simplemente que gracias a Watt consumimos watts. Cada vez más kilowatts por hora. La capacidad de generar y acumular energía para su uso industrial y posteriormente residencial, la evolución hacia una baja de los costos y el aumento de confort han convertido a los últimos 300 años en una constante evolutiva desde el punto de vista del hábitat, y generó en consecuencia la proyectualidad asociada. 


\section{El diseño}

Se definirá entonces el enfoque de este texto con una mirada desde el diseño industrial, en un enfoque histórico-cultural aplicado a escala edilicia dentro de la vivienda particular. Una escala de hábitat personal, individual en lo específico, pero multiplicado por toda la población. La incidencia del diseño industrial se percibe en la masividad.

En la investigación de mi tesis doctoral (Neumarkt, 2014/17) se analiza con profundidad el producto: plancha SIAM Futura. Su diseño, su diseñador, la empresa productora, su modo de producirse, su incidencia en la sociedad, sus valores funcionales y también semánticos. Su notable publicidad y la comunicación visual. Demuestra ser uno de los casos -que la tesis denomina como: vías proyectuales- que definen la profesión en Argentina. Ya hay rastros de objetos de planchar en China en el siglo IV, cuya función era poder alisar la tela de seda. Objetos de uso manual, algunos para calentar al fuego, otros con contenedores de brasas calientes. En todos los casos con un mango. Hubo de latón, de piedra, de hierro, de mármol. La fuente calórica transmite a través del material. El calor y la presión del peso del objeto acomodan las fibras textiles. Con variantes dentro del mismo principio la plancha siguió su evolución hasta entrado el siglo veinte. Dieciséis siglos consumiendo y quemando carbón para obtener telas aplanadas. Luego se sucedieron sistemas con agua caliente, gas, alcohol y bencina en una carrera por mejorar rendimientos, pero realmente se logró un producto práctico y muy masivo cuando la electricidad llegó a los hogares. En 1882 se patentó la primera plancha eléctrica en Nueva York, pero hubo que esperar a que se difunda la red eléctrica hogareña para que el producto triunfara masivamente. Para 1924 ya estaba ampliamente establecida y perfeccionada con un termostato. El poder del sistema industrial avanzaba sin detenerse en el impacto ambiental: "( ) usando recursos del pasado (no renovable) como energía casi instantánea” (Evans, 2017).

Muy poco tiempo después, en Argentina, sucedía lo mismo. Torcuato Di Tella (padre) ya había constituido y consolidado su empresa SIAM y la producción seriada de productos bajo licencia Westinghouse era masiva. De la misma forma los gobiernos sucesivos desde 1945 habían generado políticas de ascenso social. Una década después, el consumo de productos hogareños vinculados al confort estaba establecido. No se hablaba de impacto ambiental, ni de huella de calor. Todo era simplemente progreso y como consecuencia un aumento exponencial del uso de la energía.

En 1963 la empresa SIAM Di Tella contrata a Frank Memelsdorff para generar diseño local y no depender de las licencias. Como se mencionó antes, la tesis doctoral Industria Argentina $y$ Diseño (Neumarkt, 2014/17) lo tiene al diseñador mencionado como uno de los protagonistas. Sin embargo, muy pocos del ambiente académico lo nombran. La tesis lo puso como uno de los referentes históricos, junto a Hugo Kogan y Ricardo Blanco. Y, se complementaría mencionar en este caso, la presencia de Tomás Maldonado, por su aporte teórico y de institucionalización internacional de la disciplina. Hay una referencia al trabajo de Memelsdorff en SIAM en dos publicaciones: una de época, la revista profesional Summa (Méndez Mosquera, 1963) y otra en Crónicas del diseño industrial en Argentina (Blanco, 2005).

Memelsdorff, nacido en Berlín, Alemania y emigrado al sur del Gran Buenos Aires en 1937 a los siete años de edad, tenía buenas habilidades para el dibujo y en los veranos de su adolescencia integraba un grupo de andinistas. Se convirtió por su accionar en uno de 
los primeros cartógrafos de alta montaña que hubo en el país. Como consecuencia de estas actividades fundó junto a otros inmigrantes europeos el Club Andino de Buenos Aires. Dice Sarason, cuando se refiere al interés y el talento:

En las artes de representación, el interés y el deseo de convertirse en artista suelen manifestarse a temprana edad, por lo general antes de la adolescencia. Perfectamente es posible reemplazar las palabras artes de representación por dibujo o diseño y la palabra artista por diseñador (Sarason, 2002).

Memelsdorff fue el diseñador contratado por el ingeniero Guido Di Tella (quien asumió el cargo de presidente del holding SIAM tras la muerte de su padre) para conformar el departamento de diseño de productos industriales de la empresa. Coincidía el momento con esa primera mitad de los años sesenta del siglo veinte en que la reorganización de la empresa se profundizó. Dentro de estos tecnócratas, Frank -cuya carrera de base era la Ingeniería- supo asumir el importante rol de Director de Diseño (Cochran y Reina 1962). La combinación de su pasado como cartógrafo con su formación técnico-profesional lo transformó en un diseñador idóneo para la tarea. Vale aclarar que la formación en Diseño Industrial dentro de la Universidad estaba aún en proyecto y recién para 1963 fueron creadas las carreras en la Universidad Nacional de La Plata y en la Universidad Nacional de Cuyo, donde comenzó a haber profesionales egresados a partir de 1968. (Devalle, 2009). Una entrevista realizada a Memelsdorff, durante una mañana de un incipiente otoño de 2014, en un bar de una esquina del barrio de Palermo, permitió conocer e indagar su historia. Sorprendía su destreza al caminar a pesar de su avanzada edad. Rondaría los ochenta y tres años. Su hablar era mucho más pausado, pero su memoria intacta. La entrevista -de unas dos horas de duración- y las fotografías se realizaron con mucha naturalidad. A pesar de la diferencia generacional con el entrevistado fue una conversación entre diseñadores. Durante el año 1963, como se cuenta en El Diseño Gráfico Argentino en el siglo XX (Méndez Mosquera, 2015) a instancias de un llamado del Arq. Poyard y del Ing. Guido Di Tella, Carlos Méndez Mosquera asume la reorganización de Agens, la agencia de publicidad del holding SIAM que había sido creada dos años antes. Por Agens pasaron diseñadores de la talla de Guillermo González Ruiz, Ronald Shakespear, Nicolás Jiménez y Rubén Fontana. Dos años antes, en 1961, bajo la dirección de Jorge Romero Brest, se había creado el Centro de Artes Visuales del Instituto Di Tella, con la figura relevante en el área de diseño de Juan Carlos Distéfano y por donde puede marcarse el comienzo una renovación del diseño gráfico argentino.

Fue también en ese año 1963 que Méndez Mosquera crea la revista Summa y, como se mencionó antes, las Universidades Nacionales de La Plata y Cuyo abren las dos primeras carreras de Diseño en Argentina (en las facultades de Artes de ambas universidades). También se realiza la $1^{\text {a }}$ Exposición Internacional de Diseño Industrial de Buenos Aires y se dictan en la ciudad de Buenos Aires los seminarios del diseñador finlandés Ilmari Tappiovaara y del presidente del ICSID, el británico Misha Black.

Summa, una revista profesional, que posiciona al diseño junto a la arquitectura y a la tecnología, dedica en su segundo número unas 29 páginas al Diseño Industrial y, específicamente, dos páginas completas a una plancha. La SIAM Futura en primer plano. 

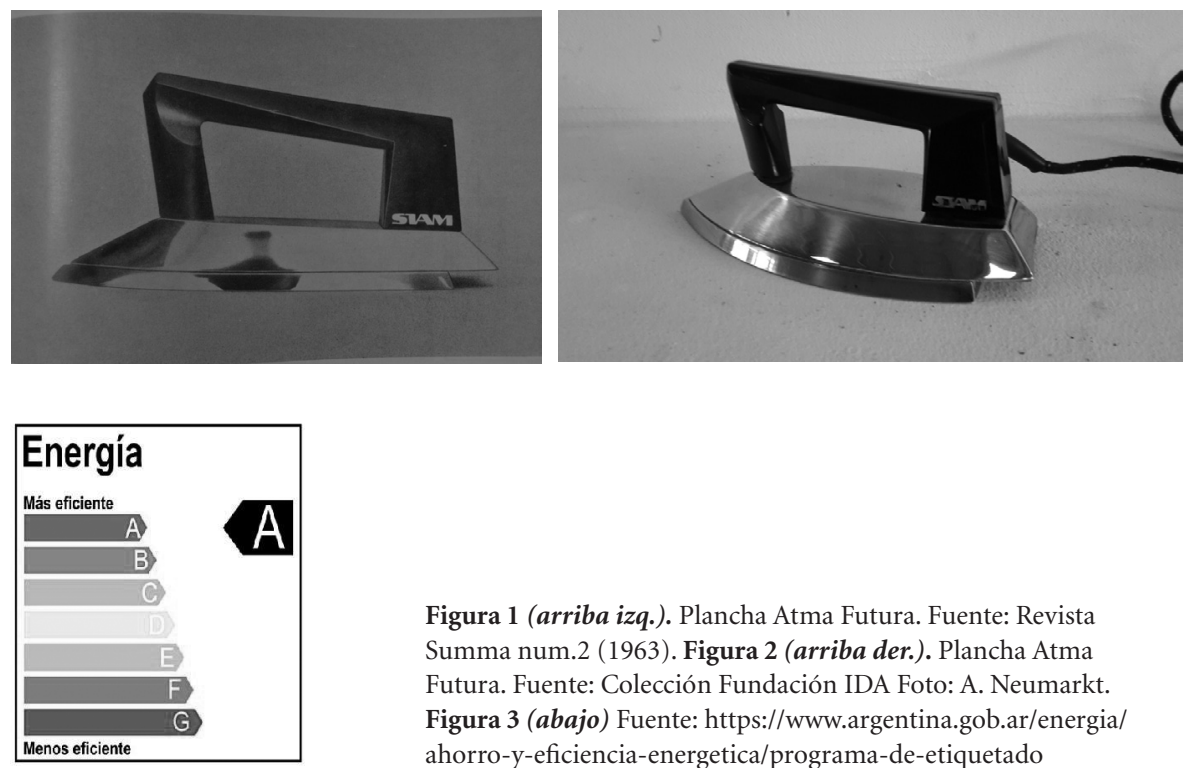

Figura 1 (arriba izq.). Plancha Atma Futura. Fuente: Revista Summa num.2 (1963). Figura 2 (arriba der.). Plancha Atma Futura. Fuente: Colección Fundación IDA Foto: A. Neumarkt. Figura 3 (abajo) Fuente: https://www.argentina.gob.ar/energia/ ahorro-y-eficiencia-energetica/programa-de-etiquetado

Este producto marca un hito en la breve historia del Diseño Industrial Argentino. Pone al diseñador en un lugar institucional dentro del sistema de poder industrial; y frente a esa tensión la empresa responde. El diseño concretiza su lugar y se define a sí mismo (Maldonado, 1977). El producto condiciona al usuario a la Modernidad, marca un antes y un después. Y la empresa SIAM, símbolo de industria nacional, representa al capitalismo como ideología racional instituida e indiscutible (Castoriadis, 2005; Schvarzer, 2006).

Como dijo el Arq. Méndez Mosquera, Director General de la revista Summa, en el prólogo a ese número dos: "Es necesario crear nuevas formas para tiempos nuevos" (1963).

Esa idea de progreso, industria, diseñador y diseño reconocido, producto de mercado masivo y la agencia comunicando, hacen de este caso, la Futura, una suma total.

Esta visión de mediados del siglo veinte, donde progreso y consumo van asociados, no consideraba la sustentabilidad. Se consume energía, se consumen productos, la industria genera trabajo, el trabajo genera consumo. Se desarrollan una profesión -el Diseño Industrial- sin cuestionamientos sobre cómo sobrevivir ecológicamente.

Si se transpolan los parámetros actuales sobre Sustentabilidad (De Schiller, Evans, 2017) al mundo industrial, queda en evidencia que en el Diseño Industrial no se ha reflexionado lo suficiente con respecto a la relación calidad de vida/consumo. Una etiqueta que indica la eficiencia energética -como traen algunos productos- es apenas un paso, pero no parece ser suficiente (Figura 3). 


\section{El presente}

Cincuenta años después de incorporar sistemáticamente electrodomésticos -que en sí mismos generan producción, empleo, diseño y calidad de vida- pero que medidos en generación de GEI (gas efecto invernadero) producen cerca del 4\% del mismo (Evans, 2017), parece necesario plantear a partir del presente siglo que, ha llegado el momento de reconsiderar la relación usuario/arquitectura/instalaciones. El impacto ambiental obviamente es mayor en rubros hogareños como calefacción y uso de agua caliente, pero más difíciles de disminuir por su mejora en la habitabilidad. Pero planchar, como acción humana, cotidiana y cultural, no genera más que gasto energético con el único beneficio de tener ropa sin arrugas.

El diseño industrial podrá perfeccionar el aparato, la tecnología podrá hacerlo más eficiente, se podrá tener materiales de mejor conductividad térmica, y otras apreciaciones, pero la pregunta a la cual no se debe arrugar, la pregunta que la disciplina debe hacerse, es la anterior a todo esto: cuál es la necesidad de que las telas no tengan pliegues. Dicho genéricamente, cuál es la necesidad, la razón de existir, de algunos productos.

La demanda promedio de consumo es $172 \mathrm{~kW} / \mathrm{h} / \mathrm{m} 2$.año (Evans, 2009). Y aunque alcance la producción actual de energía para abastecerla, toda mejora en bajar el consumo será bienvenida desde el punto de vista de la sustentabilidad. La industria podrá generar otros productos en remplazo y mantener la generación de empleo. Y el diseño industrial podrá resolver otras necesidades.

Podría suponerse que una plancha no incidiría mucho en el hogar, apenas un kW/h menos por día; pero este ahorro no debe verse como un suceso individual; solamente en la CABA hay 850.000 viviendas y casi 14.000 .000 en todo el país (INDEC, Censo 2010). Muy probablemente con una plancha en uso en un altísimo porcentaje de ellas. Y una cultura de planchado, que aunque en remisión, continúa muy establecida. Solamente suprimiendo ese objeto se obtendría un ahorro de millones de watts. Ninguna industria produce o importa lo que no se vende. En estos momentos hay más de diez modelos de planchas en venta en la red comercial. Un cambio cultural influiría directamente en la industria y en la sociedad.

Se podría plantear una legislación o normativa sobre el objeto, limitando su consumo. La SIAM Futura 1963 consume 750W. Cualquier plancha actual supera ese valor.

Se podría educar en una cultura de buenas prácticas, por ejemplo con una campaña que concentre el horario de uso en momentos de baja del consumo eléctrico.

La tecnología textil podría dar su aporte. Recrear el washewwear. Incentivar el desarrollo de telas inteligentes. Y hasta desde la moda podría plantearse lo arrugado como proyectualidad.

Una agenda verde, desde el diseño industrial, no resolvería todos los problemas, pero daría su aporte, sumaría, daría beneficios por su escala masiva, por su incidencia directa hogareña y por su capacidad de generar cultura.

El dilema del Diseño Industrial está en su origen y en su relación directa como "engranaje" de la maquinaria industrial. El ejemplo analizado es solamente referencia de toda una época y una política donde Progreso implicaba consumo, energético y económico. La profesión podrá intentar ir hacia un camino de sustentabilidad, pero deberá hacerlo en 
paralelo al de la propia industria, o no tendrá poder de imponerse. Estamos amenazados por nuestra propia tecnología, plantea Feenberg. El Diseño Industrial debe generar su propia respuesta.

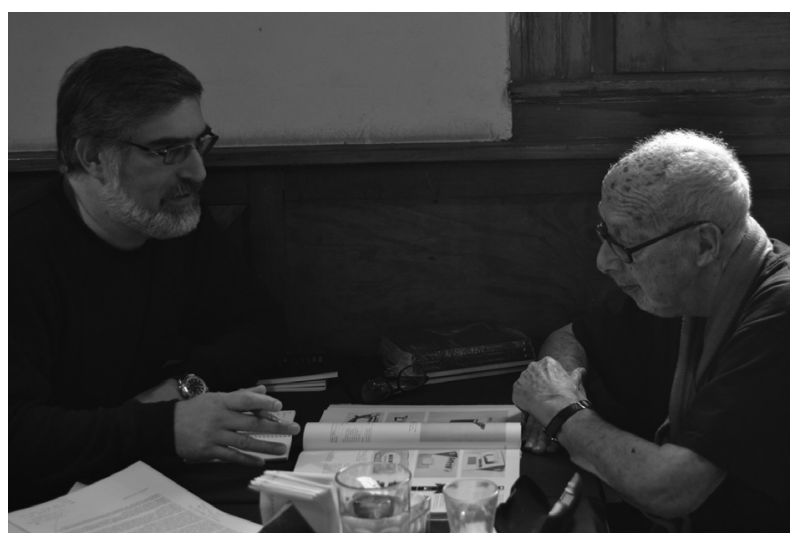

Figura 4. Frank Memmelsdorff entrevistado por Alan Neumarkt. Fuente: Julieta Nuntempuk (Junio de 2014. Bs. As. )

\section{Listado de referencias bibliográficas}

Ashton, T. (1959). La Revolución Industrial. México: Fondo de Cultura Económica.

Blanco, R. (2005). Crónicas del diseño industrial en Argentina. Buenos Aires:Ediciones FADU. UBA.

Castoriadis, C. (2005). Figuras de lo pensable. Buenos Aires: Fondo de Cultura Económica. Cochran, T. y Reina, R. (1962). Torcuato Di Tella y SIAM. Buenos Aires: Lenguaje Claro ed. / Univ. Pennsylvania Press.

Devalle, V. (2009). La travesía de la forma. Buenos Aires: Paidós.

Evans, J. (2011). El aporte del diseño en edificios energéticamente eficientes en el marco del desarrollo sustentable. Bogotá: EkoTectura.

Feenberg A. (1991). Critical Theory of Technology. Oxford: Oxford University Press. Maldonado, T. (1977). El diseño industrial reconsiderado. Barcelona: Ed. Gustavo Gili. Méndez Mosquera, C. (1963). Revista Summa num.2. Buenos Aires: Ed. Summa. Méndez Mosquera, C. (2015). Diseño gráfico argentino en el s.XX. Buenos Aires: Ed. Infinito. Neumarkt, A. (2017). Industria Argentina y Diseño, las cuatro vías proyectuales. Buenos Aires: FADU UBA (Tesis Doctoral). 
Sarason, S. (2002), La enseñanza como arte de representación. Buenos aires: Amorrortu ed.

\begin{abstract}
The dilemma of Industrial Design is in its origin and in its direct relationship as a cog in the industrial machinery. An analyzed example (the plate) serves as a reference to understand a first cultural phase, a subsequent phase of development and a recent one as a policy of progress, which implies consumption: energy and economic. Seventeen inexplicable centuries without questioning something so elementary.

The profession may try to go towards a path of sustainability, but it must do so in parallel to that of society itself, or it will not have the power to impose itself. We are threatened by our own technology, says Andrew Feenberg. Industrial Design must generate its own response.
\end{abstract}

Keywords: Design - Industry - Culture - Ecosophy.

Resumo: O dilema do Design Industrial está em sua origem e em sua relação direta como uma engrenagem na máquina industrial. Um exemplo analisado (o prato) serve como referência para entender uma primeira fase cultural, uma fase subsequente de desenvolvimento e uma recente como política de progresso, que implica consumo: energia e economia. Dezessete séculos inexplicáveis sem questionar algo tão elementar.

A profissão pode tentar seguir um caminho de sustentabilidade, mas deve fazê-lo paralelamente ao da própria sociedade, ou não terá o poder de se impor. Somos ameaçados por nossa própria tecnologia, diz Andrew Feenberg. O desenho industrial deve gerar sua própria resposta.

Palavras chave: Design - Indústria - Cultura - Ecosofia.

[Las traducciones de los abstracts fueron supervisadas por el autor de cada artículo] 